\title{
Role of Electroretinography in Investigation of Impaired Visual Function in Childhood
}

\author{
KENNETH WYBAR and BRIAN HARCOURT \\ From The Hospital for Sick Children, Great Ormond Street, London
}

Visual function is difficult to assess in young children because their limited ability to understand and communicate determines the necessity of adopting, to a large extent, objective methods of examination and investigation (Harcourt and Wybar, 1966). This also applies sometimes to older children who are not sufficiently co-operative in their subjective responses.

The afferent visual system is a complex mechanism (Fig. 1), and a dysfunction of any of its components may cause a profound visual defect. When a clinical examination of a young child suggests that the vision is defective the site of the impairment may be obvious (as in some disorder of the ocular media, like a cataract), but sometimes it is not apparent because of an absence of any objective clinical evidence of an ocular disorder. There are several reasons for this difficulty. In some cases there may be a gross functional abnormality of the retinal elements without any obvious ophthalmoscopic evidence of retinal disease, and in others there may be an optic atrophy difficult to distinguish from the physiological pallor of the optic discs commonly seen in normal young children. Furthermore, there may be a dysfunction of the optic radiation or visual cortex which is not reflected in any changes in the pupillary light reflexes or the optic discs because of the suprageniculate nature of the lesion, or the child may show little or no interest in visual stimuli simply because of a gross degree of mental retardation. Finally, an older child's inability to give a correct response in subjective visual tests may be due to a state of hysteria.

In these conditions in early childhood the normal clinical methods of examination are often inadequate for the assessment of visual function and for the localization of the visual defect so that additional forms of investigation are necessary; these include electrodiagnostic methods which are applied to the visual apparatus-electroretinography, electro-

Received May 20, 1968. oculography, and visually evoked cortical responses. In this paper only electroretinography is discussed.

When the healthy retina is stimulated by a bright flash of light a complex series of changes is induced in the electrical potential of the retinal elements. The sum of these changes may be detected by placing an active electrode on the cornea by means of a contact lens, and also earth and reference electrodes on the skin of the face (Fig. 2). When amplified and recorded these changes are summated to form the electroretinogram (ERG) which has two principal features (Karpe, 1945): an initial a-wave of negative potential and a subsequent b-wave of positive potential (Fig. 3). These electrical changes represent a mass response from all the elements in the outer retinal layers. The experimental investigation of the alterations which occur in the wave-form of this response under the influence of selective retinotoxic drugs suggests that the a-wave is due principally to changes occurring in the electrical potential of the outer segments of the retinal receptors as the result of light stimulation, and the b-wave is due principally to changes in the bipolar cells (Noell, 1959). The changes in potential which occur in the ganglion cells of the retina and in the optic nerve fibres after stimulation of the retina by light form no significant part of the ERG response, so that it is normal in cases of optic atrophy despite a profound loss of vision. The amplitude of the response is proportional to the total number of retinal elements stimulated, and because of the preponderance of the rods over the cones the total response is due principally to rod activity; this is illustrated by the increase in the amplitude of the response which follows a progressive dark adaptation because this enhances the light sensitivity and the electrical activity of the rods.

The amplitude and wave-form of the ERG response may be abnormal in several different ways; it may be inverted if only the negative a-wave is present, subnormal if both the a-wave and the b-wave are diminished in amplitude, or absent. At birth 
Fig. 1. A diagrammatic representation of the afferent pathway, demonstrating the three orders of sensory neurones involved in the relay of information between the retinal receptors (rods and cones) and the visuo-sensory cortex. (1) The retinal receptors; (2) the bipolar cells (first order neurones); (3) the optic nerve fibres, passing without delay into the optic chiasma and the optic tract (second order neurones); (4) The optic radiation (third order neurones). L.G.B. = the lateral geniculate body; V.S.C. = the visuo-sensory

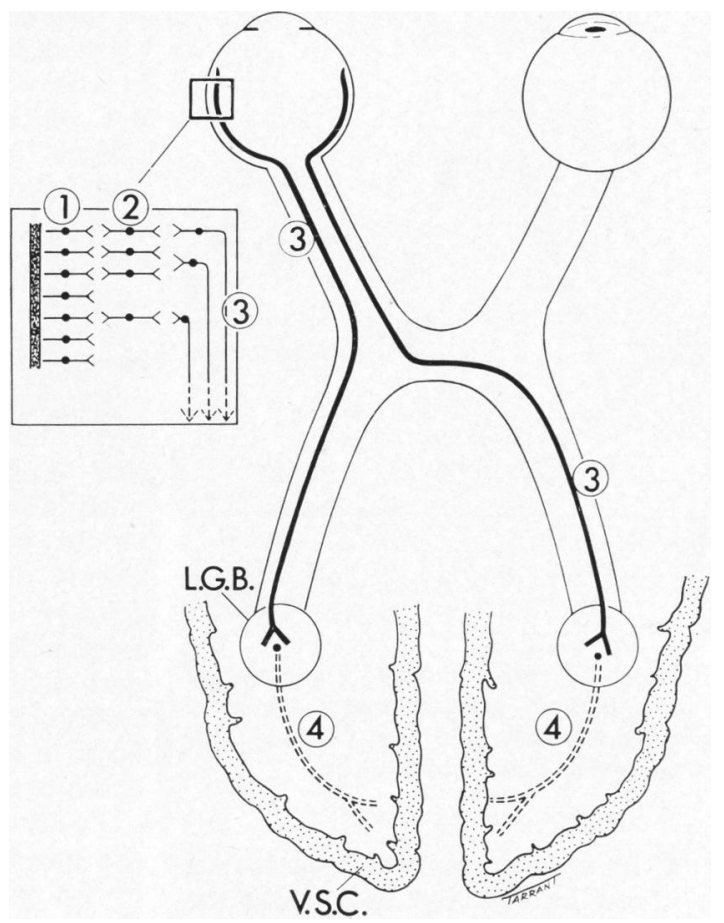
cortex.

FIG. 2. Electroretinography in a young child. The contact lens bearing the active electrode and the reference and earth electrodes have been applied under general anaesthesia. 


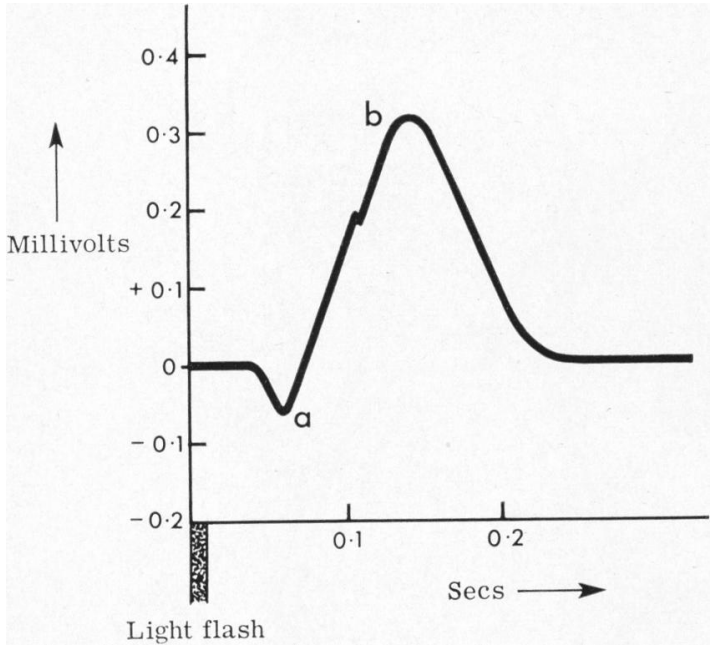

FIG. 3. A diagrammatic representation of the clinical ERG response in a normal subject showing the short latent period following the light flash, and the amplitude and wave-form of the $\mathrm{a}-$ and $\mathrm{b}-$-waves.

the amplitude of the normal response is extremely small, so that it can be detected only when the light stimulus is intense (Winkelman and Horsten, 1962). It increases gradually during the first six months of life, and thereafter it rapidly approaches adult levels. After that age an inverted, subnormal, or absent ERG response implies a widespread dysfunction of the outer retinal layers, because the mass nature of the response means that there is little or no reduction in its amplitude until about half the retina is functioning abnormally. This implies that a dysfunction of the macular area alone, while sufficient to cause a profound defect of the central vision, causes no impairment of the ERG. Furthermore, provided the light stimulus is sufficiently intense a normal ERG response is obtained in the presence of a healthy retina when there is an opacity of the ocular media that is sufficiently dense to make an ophthalmoscopic examination of the fundus impossible, and to give rise to a marked visual defect.

The practical technique of this investigation requires the insertion of a flush-fitting contact lens bearing an electrode over the cornea and the placing of skin electrodes on the face. It is essential for the eye to remain steady during the recording of the ERG, otherwise artefacts appear in the tracing because of changes in the orientation of the standing potential of the eye and because of a defective contact between the active electrode in the contact lens and the cornea. This is achieved in adults and in older children by a voluntary effort to keep the eyes steady or by the maintenance of a steady fixation of a dim source of light by the eye which is not under examination, but this is seldom possible in young children because of their natural apprehension and because of the slight discomfort which is caused by the insertion of the contact lens. It follows that the investigation requires general anaesthesia in children under the age of 6 years, and sometimes in older children who are nervous or mentally retarded. The investigation under general anaesthesia can be combined with a thorough examination of the ocular media and fundi, and with a photographic recording of any abnormal appearances. Preliminary dilatation of the pupils with cyclopentolate $1 \%$ drops is carried out and the ERG response in the lightadapted state is recorded. The increase in the amplitude of the response which occurs during dark adaptation is then studied, and this creates additional problems in the anaesthetized child because it is not possible for the anaesthetist to observe the child adequately in darkness. This difficulty cannot be overcome by simply padding the eyes to exclude the light because the pressure of the padding is likely to disturb the position of the corneal contact lens, and we have, therefore, adopted a technique of surrounding the child's head with a cage covered with an opaque black material so that all light is excluded from the eyes without affecting the illumination of the examination room and of the rest of the child's body. Repeated recordings may then be made using a light flash source incorporated within the cage (Fig. 4).

The value of the ERG is illustrated in an assessment of the hereditary tapeto-retinal degenerations. These disorders are readily diagnosed on ophthalmoscopic examination when they present with the typical features of a retinitis pigmentosa-bone corpuscle retinal pigmentation, attenuated retinal arteries, and pale optic discs. Sometimes, however, in the congenital form, the so-called Leber's congenital amaurosis (Leber, 1869), or in the juvenile form, there may be little or no abnormality of the fundi in the early stages despite a gross visual impairment or even blindness. These forms of tapeto-retinal degeneration occur more frequently than is generally recognized, and the ERG is of critical importance in establishing the diagnosis because the diminution or extinction of the ERG response reflects the widespread retinal dysfunction. The ERG also differentiates a visual defect due to a tapeto-retinal degeneration from one due to a disorder of the higher visual pathways (which 


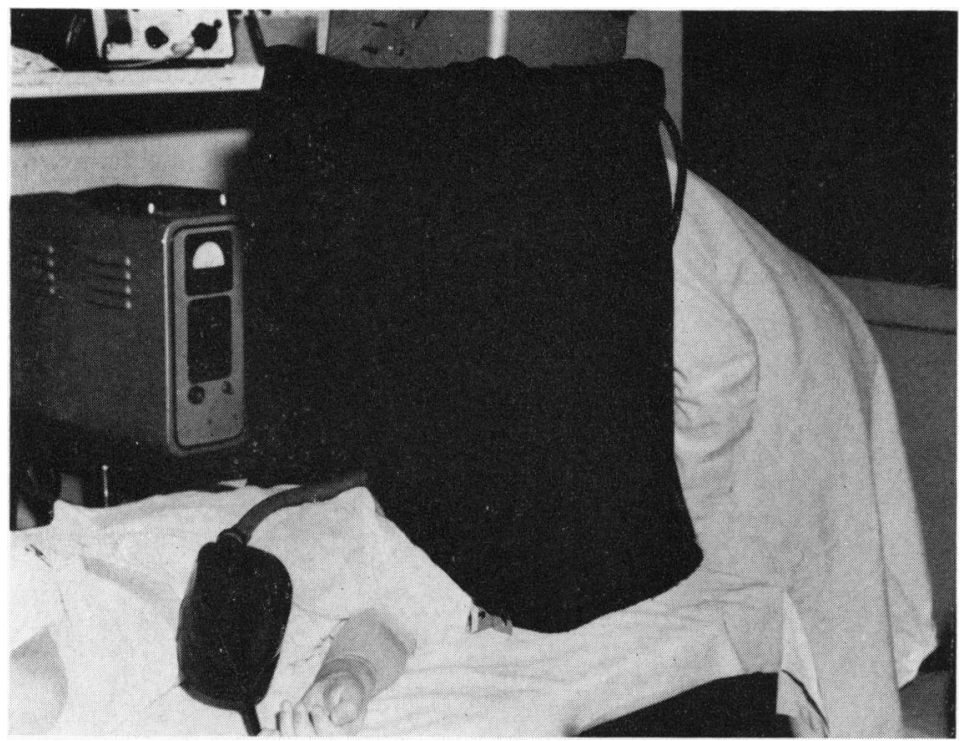

FIG. 4. Electroretinography under general anaesthesia. The child's head is surrounded by an opaque cage in which is incorporated the light flash source. The eyes are dark adapted despite the normal illumination of the examination room.

also seldom show any abnormality of the fundi), because the latter has a normal ERG.

The following case reports demonstrate the value of the ERG.

\section{Case Reports}

Case 1. This girl was born in August 1966 of unrelated parents. Her mother's pregnancy had been uneventful and delivery was normal. She was referred at the age of 4 months because the parents suspected that her vision was defective. On examination no responses were elicited to moving lights or targets, and the pupillary light reflexes were noted to be inconsistent and sluggish. The child was admitted to The Hospital for Sick Children, Great Ormond Street, for a general investigation, and her mental and physical development was found to be within normal limits for her age. Photic stimulation during the recording of the standard electroencephalogram (EEG) revealed no abnormality. The eyes were examined under general anaesthesia, and the fundi showed marked tigroid features with some scattered pigment disturbance, but there was no retinal arterial attenuation or optic atrophy. The ERG was absent in each eye.

The child's condition was reviewed at the age of 8 months. There were marked roving eye movements and a variable alternating divergent squint. No ocular fixation or following responses could be elicited using moving targets; the pupillary light reflexes were sluggish and an ophthalmoscopic examination revealed some indefinite attenuation of the retinal arteries. At the age of 14 months there were still no responses to visual stimuli and ophthalmoscopic examination showed pathological alterations in the retinal pigment pattern, particularly in the macular regions of both eyes, with retinal arterial attenuation and some optic atrophy. A very small inverted ERG response was obtained with an intense light stimulus. A diagnosis of Leber's congenital amaurosis (congenital tapeto-retinal degeneration) was made. There was no family history of a similar condition.

Case 2. This female child was born in August 1959 of unrelated parents. She developed whooping cough at the age of 2 months, and from that time her parents noticed a marked unsteadiness of her eyes and poor responses to visual stimuli. At the age of 10 months the child was examined at The Hospital for Sick Children, Great Ormond Street. She was found to have a coarse, rapid, pendular nystagmus in all directions of gaze and poor ocular fixation and following responses. Ophthalmoscopy revealed no abnormality, and a diagnosis of idiopathic congenital nystagmus was made. Despite a considerable visual handicap, the child was able to attend a normal school and at the age of 7 years her visual acuity using a telescopic visual aid was $3 / 36$ for distant and N 10 for near fixation with each eye. The nystagmus at that stage was unchanged in character and was associated with a variable alternating divergent squint. The pupillary light reflexes were rather sluggish but the fundi were still normal in appearance, apart from a very slight attenuation of the retinal arteries. The ERG showed an absence of any recordable response from either eye. A diagnosis of congenital tapetoretinal degeneration was made. The patient's elder brother had normal vision, but her younger brother was found to suffer from a similar disorder when he was brought to the hospital in 1964 at the age of 6 months. 


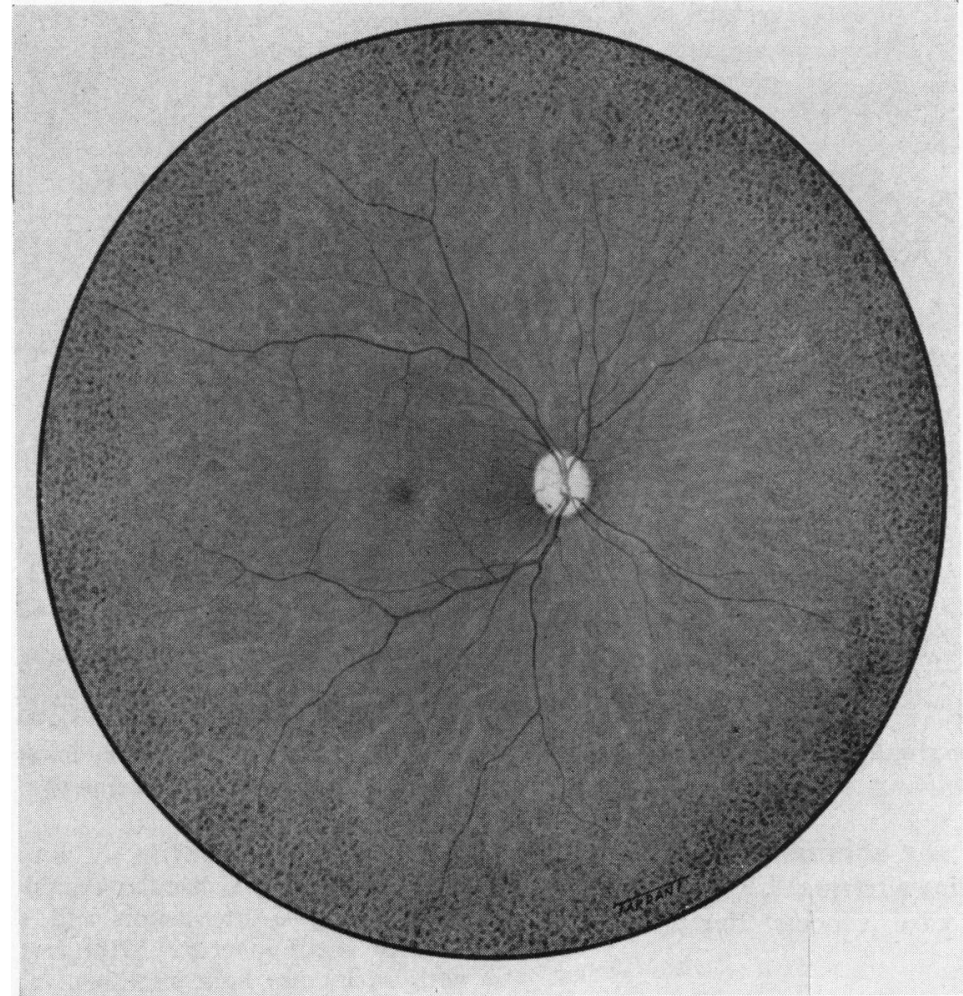

FIG. 5. Juvenile tapeto-retinal degeneration. A drawing of the right fundus of Case 3 at the age of 10 years. The pathological features are a pallor of the optic disc, marked attenuation of the retinal arteries, and in the retinal periphery an abnormal stippled pigmentation.

Case 3. This male child was born in November 1957 of unrelated parents. When he was 6 years old his parents and teachers first suspected that his vision was abnormal, and at the same time he began to have difficulty in concentrating at school, appearing to 'daydream' much of the time. He was referred to another hospital where it was found impossible to make any accurate subjective assessment of his visual acuity. No abnormality was noted on examination of the ocular media and fundi, and in view of his highly emotional behaviour it was suspected that his visual symptoms were hysterical in origin and he was referred for a psychiatric opinion. During the next year he developed further symptoms which suggested a real visual deficit, holding reading matter very close to his face and later appearing to gaze over the top of objects which he was attempting to fix. His mother also noticed that his visual difficulties were particularly severe in conditions of dim illumination. He was referred to The Hospital for Sick Children in January 1966 for a neurological opinion. At that time the vision in each eye was assessed as $6 / 9$ using the ' $E$ ' test, but there was found to be a considerable constriction of the peripheral visual fields on confrontation testing. In addition a tendency to an eccentric type of fixation was noted with the projection of the image of the object of regard on to an area of the retina above the fovea. The ocular media and fundi were normal in appearance apart from some attenuation of the retinal arteries and a slight pallor of the optic discs. The ERG was carried out without sedation and no responses were elicted even when using very intense light stimuli. A diagnosis of juvenile tapeto-retinal degeneration was made. Since then the child's visual acuity has deteriorated very markedly, and in September 1967 it became only counting fingers; by this time there were ophthalmoscopic signs of a tapeto-retinal degeneration but not in proportion to the gross visual deficit.

Case 4. This girl was first seen in December 1964 when she was 7 years of age, because of an impairment of her vision which had been suspected when she failed to pick up objects from the floor as quickly as her younger brother. Her distant vision in each eye was reduced to $6 / 18$, but the close reading vision was normal (N 5) in each eye though this was achieved with some difficulty particularly with the right eye. She was found to have a diffuse pigmentary disturbance of the macular area of each eye, with an exposure of some of the underlying choroidal vessels, suggesting some form of macular dystrophy, and in other respects the 
fundi were normal and there was no defect of the peripheral visual fields.

She was seen again three years later, and there were then signs of a widespread disturbance of the retina, with a generalized attenuation of the retinal arteries and an accumulation of scattered pigmentary deposits in the equatorial and peripheral parts of each retina, quite apart from an increase in the macular degenerative changes. The corrected vision of the right eye was $6 / 24$ and $N 8$, and of the left eye 'counting fingers' and $\mathrm{N}$ 18. It was evident that she had a widespread tapeto-retinal degeneration, and this was confirmed during an examination under general anaesthesia by the finding of an ERG response of extremely low amplitude in each eye. A further point of interest in this case is the occurrence of a widespread tapetoretinal degeneration in a cousin (the daughter of her mother's brother who married a cousin of her father).

\section{Discussion}

The cases described confirm the value of electroretinography as an objective method of investigation which demonstrates quite clearly that the cause of the defective vision is a widespread retinal dysfunction. In all the cases there was an indication of a likely visual impairment; a poor visual response, an unsteady ocular fixation, a pendular nystagmus, or roving eye movements. But there was nothing to confirm this on straightforward examination such as ophthalmoscopy or retinoscopy except for an impairment of the pupillary light reflexes. This accounted for the difficulty in reaching a precise diagnosis on clinical grounds alone, so that the preliminary diagnosis was sometimes at fault. For example, Case 2 was thought initially to be simply a congenital idiopathic nystagmus because nystagmus was the predominant symptom, and Case 3 was considered for a time to be a case of hysteria because of the delayed and insidious onset of the visual symptoms which were superimposed on a considerable emotional disturbance. Case 4 illustrates a slightly different problem as the initial symptoms suggested a visual defect affecting only the central portion of the visual field so that a macular dystrophy was a reasonable assumption, but the near extinction of the ERG responses at a later stage indicated that the retinal abiotrophic process was much more profound and widespread than was initially apparent on clinical examination. This emphasizes the fact that a tapeto-retinal degeneration in children may involve the central part of the retina before showing any obvious signs of an involvement of the peripheral parts; it is likely, however, that even in the early stages the electroretinogram provides an indication of the more widespread nature of the disorder, and it is unwise to regard a macular dystrophy in a child as an isolated event without carrying out this investigation.

Though, unhappily, it is at the present time impossible to offer any specific treatment for the arrest or reversal of tapeto-retinal degenerative changes, it is of great importance that their presence, severity, and extent should be determined at the earliest opportunity, because the distinction between congenital idiopathic nystagmus, macular dystrophy, and congenital or juvenile retinitis pigmentosa allows an accurate long-term visual prognosis to be made in early childhood, and also gives valuable information in genetic counselling. A child suffering from an idiopathic nystagmus in the absence of any retinal abnormality is likely to be capable of education at a normal school because, despite the impaired distant visual acuity, the close reading vision is usually good and the condition is stationary or may even tend to improve with age (Wybar, 1968). In contrast, as the degenerative process is progressive, a tapeto-retinal degeneration which clinically may resemble an idiopathic nystagmus in early childhood carries a much worse long-term prognosis so that there is an ever-increasing visual handicap, and education at a school for the partially sighted or blind is usually necessary. Similarly, a retinal dysfunction limited to the macular area alone carries a much better prognosis than a dysfunction affecting the whole retina, because the retention of peripheral visual function permits a considerable degree of visual independence, and also allows reasonably good reading vision when a telescopic visual aid is used which magnifies print sufficiently to allow the retinal image to fall outside the defective macular area. Congenital and juvenile forms of tapeto-retinal degeneration are usually inherited as autosomal recessive traits, and an early diagnosis of the disorder allows the parents to have the benefit of genetic counselling at a stage when they are likely to consider having other children.

It should be noted that electroretinography is of great value also in children who are suspected of having a tapeto-retinal degeneration because of an obvious pigmentary disturbance in the fundi. There are several conditions, quite apart from retinitis pigmentosa, which cause a pigmentary retinopathy in childhood, notably rubella retinopathy, syphilitic chorioretinitis, post-traumatic retinal pigmentation, and toxic retinopathy, but an early extinction of the ERG response occurs only in retinitis pigmentosa. Furthermore, a tapetoretinal degeneration in a child is not always an isolated event, so that its accurate determination may reveal the pattern of other abnormalities, 
particularly in such hereditary disorders as Hallgren's syndrome, Cockayne's syndrome, and Refsum's disease, in which retinitis pigmentosa is only one aspect of a widespread degenerative process.

Electroretinography is limited in its potential as a diagnostic tool because it gives information only about the function of the retinal part of the afferent visual pathway. But other forms of electrodiagnostic investigation are becoming available which show promise of yielding additional information about the function of other portions of the afferent visual system, particularly the recording of visually evoked cortical responses, which forms part of our electro-diagnostic assessment of visual defects in young children though it is outside the scope of this paper.

\section{Conclusion}

When there is apparent clinical evidence of impaired visual function in a young child, in the absence of any obvious signs of an ocular abnormality, objective methods of investigation are necessary in order to confirm the impairment and to localize its cause, or in order to refute the suggestion of an impairment. Electroretinography determines the cases in which the visual defect is due to a widespread retinal dysfunction.

\section{Summary}

The physiological basis and the practical technique of electroretinography as applied to young children are described. Four cases are reported which illustrate the value of this objective method of investigation in the diagnosis of congenital and juvenile forms of tapeto-retinal degeneration, which in their early stages may produce a widespread functional abnormality of the retinal receptors without any obvious signs of retinal disease on ophthalmoscopic examination. This investigation is also useful in distinguishing between primary and secondary pigmentary retinopathies in childhood, and in drawing attention to a tapeto-retinal dysfunction which may be only one aspect of a widespread hereditary degenerative disorder. The study of visually evoked cortical responses is another form of electrodiagnostic investigation which yields additional information about the functional integrity of the visual pathways, but the details of this method are not included in the present discussion.

We should like to thank Dr. G. Pampiglione, Dr. Geoffrey Arden, and Miss Joan Behrman for their invaluable help in making possible the electrodiagnostic parts of these investigations. We should also like to thank Dr. John Wilson for allowing us to publish Case 3 which we examined initially during admission to the Neurological Unit of The Hospital for Sick Children, Great Ormond Street.

The work of one of us (B.H.) as Research Fellow in Paediatric Ophthalmology is sponsored by a grant which has been made available to the Institute of Ophthalmology by the Variety Club of Great Britain; grateful acknowledgement is made of this.

The electrodiagnostic instruments have been made available to us by the generosity of a grant from the People Newspaper for research into 'Blindness in Infancy'.

This work has been supported by a generous grant from a gentleman who wishes to remain anonymous, and a grant from the Forest of Arden Lodge, in memory of the sacrifice of the children of Aberfan.

Thanks are due to the Institute of Child Health for help with these grants, and to Mr. T. Tarrant of the Medical Illustration Department of the Institute of Ophthalmology for preparing the fundus painting shown in Fig. 5.

\section{REFERENCES}

Harcourt, R. B., and Wybar, K. (1966). The determination of blindness in infancy. Trans. ophthal. Soc. U.K., 86, 37.

Karpe, G. (1945). The basis of clinical electroretinography. Acta ophthal.' Kbh.), Suppl. 24.

Leber, T. (1869). Ueber Retinitis pigmentosa und angeborene Amaurose. Albrecht. v. Graefes Arch. Ophthal., 15, (3), 1.

Noell, W. K. (1959). The visual cell: electric and metabolic manifestations of its life processes. Amer. F. Ophthal., 48, Suppl. 347.

Winkelman, J. E., and Horsten, G. P. M. (1962). The ERG of premature and full-term born infants during their first days of life. Ophthalmologica (Basel), 143, 92.

Wybar, K. C. (1968). Nystagmus in childhood. In Transactions of the First International Congress of Orthoptists, p. 249. Kimpton, London. 